\title{
Bounds on the Truncation Error by Finite Differences for the Goursat Problem
}

\author{
By A. K. Aziz and B. E. Hubbard
}

1. Introduction. A common method for obtaining an approximate solution to the various boundary value problems for partial differential equations is by finite differences. The fundamental problem concerning such finite difference approximations is to show that the truncation error (i.e., the difference between the exact solution and the solution of the approximating finite difference problem) tends to zero with diminishing mesh size. Further the success of a finite difference method is related to the possibility of deducing explicit a priori bounds for the solution, or to the stability of the difference scheme. Various authors have studied the question of stability and a priori estimates connected with finite difference schemes concerning hyperbolic partial differential equations (see e.g. Richtmyer [14], with references).

In general the main tool used in obtaining estimates for the solution of hyperbolic equations is the energy method.

In this paper we obtain explicit bounds in terms of the data of the problem for the linear and non-linear second order partial differential equation of hyperbolic type in two independent variables, i.e. linear boundary value problem 2.1, 2.2 and non linear problem 4.1, 4.2. The present approach is based not on the energy method but on the method of majorants which is embodied in the statement of theorem 2.1 and its corollaries.

In section 2 we first define a suitable finite difference analogue of 2.1 and develop the finite difference analogue of Riemann's function and Riemann's formula for equation 2.1. Making use of this representation of the solution $U(x, y)$ of boundary value problem 2.9, 2.10 and the majorizing theorem 2.1, together with its corollaries, we obtain in section 3 explicit bounds of $O\left(h^{2}\right)$ for the truncation errors $\mid u(x, y)-$ $U(x, y)|,| u_{x}(x, y)-U(x, y)_{x}|,| u_{y}(x, y)-U(x, y)_{y}|,| u_{x y}(x, y)-U(x, y)_{X Y} \mid$, where $u$ and $U$ denote the solution of $2.1,2.2$ and 2.9, 2.10 respectively. In section 4 , employing the results and techniques of section 3 , we obtain a similar bound of $O\left(h^{2}\right)$ for the truncation error $E(x, y)$. for 4.1. Both bounds in section 3 and in section 4 are of nondecaying exponential nature and this exponential character makes our bounds rather poor when applied to problems whose solutions have decaying exponential behavior (i.e., $u_{x y}+u_{x}+u_{y}+u=0, u(x, 0)=x$, $\left.u(0, y)=y, u(x, y)=x e^{-y}+y e^{-x}\right)$.

Applying Dames [5] criterion for stability, it is easily seen that the finite difference scheme considered is unconditionally stable.

In section 5 we give a simple numerical example where the bound for the truncation error is computed.

Received June 1, 1962. Revised April 22, 1963. This research was supported by the Naval Ordnance Laboratory through the Foundational Research Program, and the Air Force Office of Scientific Research. 
2. Finite Difference Analogue. We shall pose a finite difference analogue of the characteristic boundary value problem given by

$$
\begin{gathered}
L u \equiv u_{x y}+a u_{x}+b u_{y}+c u=f \text { in } R \\
u(x, 0)=\varphi(x) \\
u(0, y)=\psi(y) \\
\varphi(0)=\psi(0)
\end{gathered}
$$

Where $R$ is the first quadrant of the $(x, y)$ plane. Let $R_{h}$ be the set of grid points $(m h, n h) ; m, n$ positive integers and let $C_{h}$ be the boundary mesh points $(m h, 0)$ and $(0, n h)$. The real number $n>0$ is called the "mesh constant". Any function $W(x, y)$ which is defined at points of $R_{h}+C_{h}$ is called a "mesh function". We assume that the domain of definition of every mesh function is extended to the points $\left(m h-\frac{1}{2} h, n h\right),\left(m h, n h-\frac{1}{2} h\right),\left(m h-\frac{1}{2} h, n h-\frac{1}{2} h\right)$ by the following rules.

$$
\begin{aligned}
W\left(m h-\frac{1}{2} h, n h\right) & =\frac{1}{2}[W(m h, n h)+W(m h-h, n h)] \\
W\left(m h, n h-\frac{1}{2} h\right) & =\frac{1}{2}[W(m h, n h)+W(m h, n h-h)] \\
W\left(m h-\frac{1}{2} h, n h-\frac{1}{2} h\right) & =\frac{1}{2}\left[W\left(m h-\frac{1}{2} h, n h\right)+W\left(m h-\frac{1}{2} h, n h-h\right)\right] \\
& =\frac{1}{2}\left[W\left(m h, n h-\frac{1}{2} h\right)+W\left(m h-h, n h-\frac{1}{2} h\right)\right] \\
& =\frac{1}{4}[W(m h, n h)+W(m h-h, n h) \\
& \quad+W(m h, n h-h)+W(m h-h, n h-h)]
\end{aligned}
$$

By defining $W(x, y)$ at such points as the averages of $W(x, y)$ at nearby points of $R_{h}+C_{h}$ we make it possible to use the finite difference equivalent of the chain rule of differentiation. The desirability of this was pointed out by B. Wendroff [20]. At points of the extended domain of definition of $W(x, y)$ we define the finite difference equivalent of partial differentiation by

$$
\begin{aligned}
& W(x, y)_{x}=h^{-1}\left[W\left(x+\frac{1}{2} h, y\right)-W\left(x-\frac{1}{2} h, y\right)\right] \\
& W(x, y)_{Y}=h^{-1}\left[W\left(x, y+\frac{1}{2} h\right)-W\left(x, y-\frac{1}{2} h\right)\right] .
\end{aligned}
$$

From (2.4) we see that

$$
\begin{aligned}
W(x, y)_{X Y}= & W(x, y)_{Y X}=h^{-1}\left[W\left(x, y+\frac{1}{2} h\right)_{X}-W\left(x, y-\frac{1}{2} h\right)_{X}\right] \\
= & h^{-1}\left[W\left(x+\frac{1}{2} h, y\right)_{Y}-W\left(x-\frac{1}{2} h, y\right)_{Y}\right] \\
= & h^{-2}\left[W\left(x+\frac{1}{2} h, y+\frac{1}{2} h\right)-W\left(x+\frac{1}{2} h, y-\frac{1}{2} h\right)\right. \\
& \left.\quad-W\left(x-\frac{1}{2} h, y+\frac{1}{2} h\right)+W\left(x-\frac{1}{2} h, y-\frac{1}{2} h\right)\right] .
\end{aligned}
$$

We note that for the mesh functions $V(x, y), W(x, y)$ and $m, n$ integers we have

$$
\begin{aligned}
& {\left[V\left(m h-\frac{1}{2} h, y\right) W\left(m h-\frac{1}{2} h, y\right)\right]_{X}} \\
& \quad=V\left(m h-\frac{1}{2} h, y\right) W\left(m h-\frac{1}{2} h, y\right)_{X}+V\left(m h-\frac{1}{2} h, y\right)_{X} W\left(m h-\frac{1}{2} h, y\right) \\
& {\left[V\left(x, n h-\frac{1}{2} h\right) W\left(x, n h-\frac{1}{2} h\right)\right]_{Y}} \\
& \quad=V\left(x, n h-\frac{1}{2} h\right) W\left(x, n h-\frac{1}{2} h\right)_{Y}+V\left(x, n h-\frac{1}{2} h\right)_{Y} W\left(x, n h-\frac{1}{2} h\right)
\end{aligned}
$$


Let the mesh functions $A$ and $B$ be given at points $\left(x+\frac{1}{2} h, y\right)$ and $\left(x, y+\frac{1}{2} h\right)$ respectively by

$$
\begin{aligned}
& A\left(x+\frac{1}{2} h, y\right)=a\left(x+\frac{1}{2} h, y\right) \\
& B\left(x, y+\frac{1}{2} h\right)=b\left(x, y+\frac{1}{2} h\right)
\end{aligned}
$$

where $x=m h-\frac{1}{2} h, y=n h-\frac{1}{2} h$. Similarly let $\Phi$ and $\Psi$ be defined on their respective subsets of $C_{h}$ as

$$
\begin{array}{ll}
\Phi(x)=\varphi(x) & x=m h \\
\Psi(y)=\psi(y) & y=n h .
\end{array}
$$

We formulate the following finite difference analogue of the characteristic boundary value problem (2.1) and (2.2).

$$
\begin{gathered}
L_{h} U \equiv U_{X Y}+A U_{X}+B U_{Y}+C U=f(x, y) \\
x=m h-\frac{1}{2} h, \quad y=n h-\frac{1}{2} h ; \quad m, n \text { positive integers, } \\
U(m h, 0)=\Phi(m h) \\
U(0, n h)=\Psi(n h) .
\end{gathered}
$$

In (2.9) $A$ and $B$ are given as averages of the values at the points of (2.7) whereas $C(x, y)$ and $f(x, y)$ are values at the point $(x, y)$. We see that $(2.9)$ and $(2.10)$ is a system of linear equations for $U(m h, n h)$ and that in each equation of (2.9) the mesh function $U(m h, n h)$ is given as a linear combination of $U(m h-h, n h-h)$, $U(m h, n h-h), U(m h-h, n h)$ and $F$. Hence (2.9) and (2.10) can be solved explicitly.

In preparation for deriving a finite difference form of Riemann's formula we now introduce a certain Riemann-Stieltjes integral as has been done in elliptic problems by G. Forsythe and W. Wasow [8]. Let the step functions $\mu(x), \nu(y)$ be defined by

$$
\begin{array}{ll}
\mu(x)=m h ; \quad & m h-\frac{1}{2} h<x \leqq m h+\frac{1}{2} h \\
\nu(y)=n h ; & n h-\frac{1}{2} h<y \leqq n h+\frac{1}{2} h .
\end{array}
$$

The Riemann-Stieltjes integral with respect to $\mu(x)$ is seen to be

$$
\int_{0}^{m h} f(x) d \mu=h \sum_{m=1}^{M} f\left(m h-\frac{1}{2} h\right) .
$$

In particular if $W(x, y)$ is a mesh function and we assume that the definition of $W(x, y)$ is extended in a smooth manner to the entire $(x, y)$ plane then we have

$$
\int_{0}^{M h} W(x, y) d \mu=h \sum_{m=1}^{M} W\left(m h-\frac{1}{2} h, y\right) .
$$

Likewise the Riemann-Stieltjes integral with respect to $\nu(y)$. is seen to be

$$
\int_{0}^{N h} W(x, y) d \nu=h \sum_{n=1}^{N} W\left(x, n h-\frac{1}{2} h\right) .
$$

Let $\xi=M h . \eta=N h ; M, N$ positive integers and assume that $W(x, y)$ and 
$V(x, y)$ are mesh functions such that $W$ vanishes at points of the $x$ and $y$ axes. From the definition of $W(x, y), V(x, y)$ in the extended domain together with the chain rule for differences (2.6) we can carry through the usual integration by parts with differentiation replaced by differencing to obtain

$$
\begin{aligned}
\int_{0}^{\xi} d \mu \int_{0}^{\eta} & d \nu V(x, y) L_{h} W(x, y)=\int_{0}^{\xi} d \mu \int_{0}^{\eta} d \nu W(x, y) L_{h}{ }^{*} V(x, y) \\
& -\int_{0}^{\xi}\left[V(x, \eta)_{x}-B(x, \eta) V(x, \eta)\right] W(x, \eta) d \mu \\
& -\int_{0}^{\eta}\left[V(\xi, y)_{Y}-A(\xi, y) V(\xi, y)\right] W(\xi, y) d \nu+V(\xi, \eta) W(\xi, \eta) .
\end{aligned}
$$

In (2.15) we have introduced the finite difference adjoint operator $L_{h}{ }^{*}$ of $L_{h}$ which is given by

$$
L_{h}^{*} V(x, y) \equiv V_{X Y}-(A V)_{X}-(B V)_{Y}+C V .
$$

We now define for each $P=(\xi, \eta)$ the mesh function $V(x, y ; P)=V(x, y ; \xi, \eta)$ to be the solution of the finite difference problem

$$
\begin{array}{lc}
L_{h}^{*} V(x, y ; P)=0 ; \quad 0<x=m h-\frac{1}{2} h<\xi \text { and } \\
\\
V(x, \eta ; P)_{x}-B(x, \eta) V(x, \eta ; P)=0 ; & 0<x=m h-\frac{1}{2} h<\xi \\
V(\xi, y ; P)_{Y}-A(\xi, y) V(\xi, y ; P)=0 ; & 0<y=n h-\frac{1}{2} h<\eta \\
V(P ; P)=1 .
\end{array}
$$

Again we observe that $V(x, y ; P)$ is given explicitly by $(2.17)$ so that there is no question as to its existence and uniqueness. Also for $V$ determined by (2.17) when substituted into (2.15) we have the finite difference form of Riemann's formula

$$
W(\xi, \eta)=\int_{0}^{\xi} d \mu \int_{0}^{\eta} d \nu V(x, y ; \xi, \eta) L_{h} W(x, y)
$$

for mesh functions $W(\xi, \eta)$ which vanish at points of the $x$-axis and the $y$-axis. If $W(P)$ does not vanish on the axes then (2.18) takes the more general form

$$
\begin{aligned}
W(\xi, \eta) & =+V(0,0 ; \xi, \eta) W(0,0) \\
& +\int_{0}^{\xi}\left[W(x, 0)_{x}-B(x, 0) W(x, 0)\right] V(x, 0) d \mu \\
& +\int_{0}^{\eta}\left[W(0, y)_{Y}-A(0, y) W(0, y)\right] V(0, y) d \nu \\
& +\int_{0}^{\xi} d \mu \int_{0}^{\eta} d \nu V(x, y ; \xi, \eta) L_{h} W(x, y) .
\end{aligned}
$$

We shall now derive some further finite difference analogues of classical results concerning the solution of (2.1), (2.2). Finally we shall conclude this section with a theorem concerning certain majorizing finite difference problems. We first give a 
finite difference form of the function $e^{f(x)}$. This function arises as a solution of the differential equation

$$
y^{\prime}(x)=f^{\prime}(x) y(x)
$$

The finite difference exponential arises as the solution of the finite difference analogue of (2.20) as given in the following lemma.

Lemma 2.1. For $K(x)$ given at $x=m h ; m=0,1, \cdots, M$ we define the mesh function $e_{h}{ }^{K(x)}$ as the solution of the difference equation

$$
\left[e_{h}{ }^{K(x)}\right]_{X}=K(x)_{X} e_{h}{ }^{K(x)} ; \quad x=\frac{1}{2} h, 3 / 2 h, \cdots,\left(M-\frac{1}{2}\right) h .
$$

In (2.21) we define

$$
\begin{aligned}
K\left(m h-\frac{1}{2} h\right) & =\frac{1}{2}[K(m h)+K(m h-h)] \\
e_{h}{ }^{K\left(m h-\frac{1}{h} h\right)} & =\frac{1}{2}\left[e_{h}{ }^{K(m h)}+e_{h}{ }^{K(m h-h)}\right] .
\end{aligned}
$$

Then for $x=m h, e_{h}{ }^{K(x)}$ is given by the formula

$$
e_{h}{ }^{K(x)}=e_{h}{ }^{K(0)} \prod_{m=1}^{M}\left[\frac{1+\frac{1}{2} h K\left(m h-\frac{1}{2} h\right)_{X}}{1-\frac{1}{2} h K\left(m h-\frac{1}{2} h\right)_{X}}\right]
$$

Proof. For fixed $m$ we solve (2.21) to obtain

$$
e_{h}{ }^{K(m h)}=e_{h}{ }^{K(m h-h)}\left[\frac{1+\frac{1}{2} h K\left(m h-\frac{1}{2} h\right)_{X}}{1-\frac{1}{2} h K\left(m h-\frac{1}{2} h\right)_{X}}\right]
$$

Solving this recursion formula we obtain (2.22).

We can apply Lemma 2.1 to the boundary conditions in (2.17) to obtain

$$
\begin{array}{ll}
V(\bar{x}, \eta ; \xi, \eta)=\exp _{h}\left[-\int_{\bar{x}}^{\xi} B(x, \eta) d \mu\right] & \bar{x} \leqq \xi \\
V(\xi, \bar{y} ; \xi, \eta)=\exp _{h}\left[-\int_{\bar{y}}^{\eta} A(\xi, y) d \nu\right] & \bar{y} \leqq \eta
\end{array}
$$

since $V(\xi, \eta ; \xi, \eta)=1$.

A comparison of the series expansion of $e^{x}$ and $e_{h}^{x}$ reveals that

$$
e^{x} \leqq e_{h}^{x} \quad x=m h \geqq 0 .
$$

We have the following inequality relating our Stieltjes integral to the Riemann integral.

LEMmA 2.2. If $f(x) \in C^{2}$ and $f^{\prime \prime}(x) \geqq 0$, then for any positive integer $m$ we have

$$
\int_{0}^{m h} f(x) d \mu \leqq \int_{0}^{m h} f(x) d x .
$$

Proof. If $x_{0}=r h-\frac{1}{2} h, r$ an integer, then

$$
f(x)=f\left(x_{0}\right)+f^{\prime}\left(x_{0}\right)\left(x-x_{0}\right)+\int_{x_{0}}^{x} f^{\prime \prime}(\xi)(x-\xi) d \xi .
$$

Hence

$$
\int_{r h-h}^{r h} f(x) d x=h f\left(x_{0}\right)+\int_{r h-h}^{r h} d x \int_{x_{0}}^{x} f^{\prime \prime}(\xi)(x-\xi) d \xi
$$


Since the second term on the right hand side of (2.27) is positive we see that

$$
\int_{r h-h}^{r h} f(x) d x \geqq h f\left(x_{0}\right)=\int_{r h-h}^{r h} f(x) d \mu .
$$

Summing the inequality (2.28) over integral values of $r$ yields (2.25).

We have the following lemma regarding differencing with respect to a parameter.

Leмma 2.3. If $W \cdot(x, y)$ is a mesh function then for $y=\cdot m h-\frac{1}{2} h$

$$
\left[\int_{0}^{y} W(x, y) d \mu\right]_{Y}=W\left(y, y+\frac{1}{2} h\right)+\int_{0}^{y-\frac{1}{2} h} W(x, y)_{Y} d \mu .
$$

Proof.

$$
\begin{array}{r}
{\left[\int_{0}^{y} W(x, y) d \mu\right]_{Y}=h^{-1} \int_{0}^{y+\frac{1}{2} h} W\left(x, y+\frac{1}{2} h\right) d \mu-\int_{0}^{y-\frac{1}{3} h} W\left(x, y-\frac{1}{2} h\right) d \mu} \\
=h^{-1} \int_{y-\frac{1}{2} h}^{y+\frac{1}{2} h} W\left(x, y+\frac{1}{2} h\right) d \mu+\int_{0}^{y-\frac{1}{2} h} W(x, y)_{Y} d \mu=W\left(y-\frac{1}{2} h, y+\frac{1}{2} h\right) \\
+\int_{0}^{y-\frac{1}{2} h} W(x, y)_{Y} d \mu .
\end{array}
$$

Since we shall be using the method of majorants to bound the "discretization error" (the difference between the solutions of (2.1) and (2.9)) the following theorem on majorizing problems for (2.9) is of central importance.

TheOREM 2.1. Let $W(x, y)$ be a solution of the finite difference problem

$$
W_{X Y}=C_{1} W_{X}+C_{2} W_{Y}+C_{3} W+C_{4}
$$

at points $\left(m h-\frac{1}{2} h, n h-\frac{1}{2} h\right)$ where the functions $C_{i}(x, y) \geqq 0$. We further assume that

$$
\begin{array}{lll}
W(x, 0) \geqq 0, & W(x, 0)_{X} \geqq 0, & x \geqq 0 \\
W(0, y) \geqq 0, & W(0, y)_{Y} \geqq 0, & y \geqq 0 .
\end{array}
$$

Finally let

$$
\left(1-\delta_{3}\right)>0, \quad\left(1-\delta_{4}\right) \geqq 0
$$

where

$$
\begin{aligned}
& \delta_{1}=\frac{1}{2} h\left(C_{1}+\frac{3 C_{3} h}{4}\right) \\
& \delta_{2}=\frac{1}{2} h\left(C_{2}+\frac{3 C_{3} h}{4}\right) \\
& \delta_{3}=\frac{1}{2} h\left(C_{1}+\frac{C_{3} h}{4}\right) \\
& \delta_{4}=\frac{1}{2} h\left(C_{2}+\frac{C_{3} h}{4}\right) \\
& \delta_{5}=h\left[C_{3} W(x, y)+C_{4}\right] .
\end{aligned}
$$


Then we conclude that

$$
W \geqq 0, \quad W_{X} \geqq 0, \quad W_{Y} \geqq 0, \quad W_{X Y} \geqq 0
$$

at points of $R_{h}$.

Proof. The theorem is proved by induction. Let $x=m h, y=n h$ be an arbitrary point of $R_{h}$. We introduce the notation

$$
\begin{gathered}
a_{1}=W(x, y)_{X}, \quad a_{2}=W(x, y+h)_{X}, \\
b_{1}=W(x, y)_{Y}, \quad b_{2}=W(x+h, y)_{Y}, \quad d_{1}=W(x, y) .
\end{gathered}
$$

Our induction assumption is that

$$
a_{1} \geqq 0, \quad b_{1} \geqq 0, \quad d_{1} \geqq 0 .
$$

We see that 2.29 can be written in the alternate forms

$$
\begin{aligned}
& a_{2}\left(1-\delta_{3}\right)=a_{1}\left(1+\delta_{1}\right)+\delta_{2} b_{1}+\delta_{4} b_{2}+\delta_{5} \\
& b_{2}\left(1-\delta_{4}\right)=b_{1}\left(1+\delta_{2}\right)+\delta_{1} a_{1}+\delta_{3} a_{2}+\delta_{5} .
\end{aligned}
$$

Solving these equations simultaneously under the assumption (2.31) we see that

$$
\begin{aligned}
a_{2}\left(1-\delta_{3}-\frac{\delta_{1} \delta_{4}}{1-\delta_{4}}\right)=a_{1} & \left(1+\delta_{1}+\frac{\delta_{1} \delta_{4}}{1-\delta_{4}}\right) \\
& +b_{1}\left[\delta_{2}+\delta_{4}\left(\frac{1+\delta_{2}}{1-\delta_{4}}\right)\right]+\delta_{5}\left(1+\frac{\delta_{4}}{1-\delta_{4}}\right) \\
b_{2}\left(1-\delta_{4}-\frac{\delta_{2} \delta_{3}}{1-\delta_{3}}\right)=b_{1} & \left(1+\delta_{2}+\frac{\delta_{2} \delta_{3}}{1-\delta_{3}}\right) \\
& +a_{1}\left[\delta_{1}+\delta_{3}\left(\frac{1+\delta_{1}}{1-\delta_{3}}\right)\right]+\delta_{5}\left(1+\frac{\delta_{3}}{1-\delta_{3}}\right) .
\end{aligned}
$$

From (2.35) and (2.34) it follows immediately that

$$
a_{2} \geqq a_{1} \geqq 0 ; \quad b_{2} \geqq b_{1} \geqq 0
$$

and hence that

$$
W(x+h, y+h) \geqq\left\{\begin{array}{l}
W(x+h, y) \geqq 0 \\
W(x, y+h) \geqq 0
\end{array}\right.
$$

Clearly then $W\left(x+\frac{1}{2} h, y+\frac{1}{2} h\right)_{X Y} \geqq 0$ and the theorem is proved.

We can obtain an explicit upper bound for $W(x, y)$ in the following special case.

CoRollary 1. Let $W(x, y)$ satisfy the hypotheses of Theorem 2.1. In addition we assume that $C_{i}$ are constants and that $W(x, 0)=W(0, y)=0$. Then $W(x, y)$ satisfies the inequality

$$
W(\xi, \eta) \leqq C_{4} \int_{0}^{\xi} d x \int_{0}^{\eta} d y\left[e_{h} \sqrt{C_{h}}\{(\xi-x)+(\eta-y)\}\right]\left[e_{h}{ }^{C_{2}(\xi-x)+C_{1}(\eta-y)}\right]
$$

where

$$
C_{5}=C_{3}+C_{1} C_{2}
$$


Proof. If we define the function $V(x, y)$ at points $x=m h, y=n h$ by the equation

$$
W(x, y)=V(x, y) e_{h}^{c_{2} x+c_{1} y}
$$

we see that $V(x, y)$ is a solution of the finite difference problem

$$
\begin{gathered}
V_{X Y}(x, y)=C_{5} V(x, y)+C_{i} e_{h}^{-\left(C_{2} x+C_{1} y\right)}, \quad x=m h-\frac{1}{2} h, y=n h-\frac{1}{2} h \\
V(x, 0)=V(0, y)=0
\end{gathered}
$$

The finite difference Riemann's function, $V^{*}(x, y ; \xi, \eta)$, related to the problem (2.39) as given in (2.17) satisfies the adjoint problem

$$
\begin{aligned}
& V^{*}(x, y ; \xi, \eta)_{X Y}=C_{5} V^{*}(x, y ; \xi, \eta) \\
& V^{*}(x, \eta ; \xi, \eta)_{X}=0 \\
& V^{*}(\xi, y ; \xi, \eta)_{Y}=0
\end{aligned}
$$

It is easily verified that

$$
V^{*}(x, y ; \xi, \eta) \leqq e_{h} \sqrt{\bar{c}_{\bullet}}[(\xi-x)+(\eta-y)]
$$

From the finite difference Riemann's formula (2.18) with $L_{h}$ given in (2.39) we see that

$$
\left.V(\xi, \eta) \leqq C_{4} \int_{0}^{\xi} d \mu \int_{0}^{\eta} d \nu\left[e_{h} \sqrt{c_{5}}(\xi-x)+(\eta-y)\right\}\right]\left[e_{h}^{-\left(c_{2} x+c_{1} y\right)}\right]
$$

The result (2.36) now follows from (2.38) and Lemma 2.2. The substitution (2.38) and the subsequent bound are motivated by analogous results for the differential equation (2.1), R. von Mises [17, p. 812].

The following corollary gives the form of the majorizing theorem which we shall utilize in obtaining bounds on the discretization error.

Corollary 2. Let the quantities in Theorem 2.1 have superscripts (1) and (2) to indicate two distinct finite difference problems. We replace the assumption $C_{i}{ }^{(1)}(x, y) \geqq 0$ by $C_{i}{ }^{(2)} \geqq\left|C_{i}{ }^{(1)}\right|$ and $(2.30)$ by $W^{(i)}(x, 0)=W^{(i)}(0, y)=0$. Then it follows that

$$
\begin{array}{ll}
W^{(2)}(x, y) \geqq\left|W^{(1)}(x, y)\right| ; & W^{(2)}(x, y)_{\mathbf{X}} \geqq\left|W^{(1)}(x, y)_{\mathbf{X}}\right| \\
W^{(2)}(x, y)_{\mathbf{Y}} \geqq\left|W^{(1)}(x, y)_{\mathbf{Y}}\right|_{;} & W^{(2)}(x, y)_{\mathbf{X} Y} \geqq\left|W^{(1)}(x, y)_{\mathbf{X}}\right|
\end{array}
$$

Proof. As in the proof of Theorem 2.1 the proof is by induction. From the hypotheses of the corollary together with (2.35) and the induction assumption we see that

$$
\begin{aligned}
&\left|a_{2}{ }^{(1)}\right| \leqq {\left[1-\left|\delta_{3}{ }^{(1)}\right|-\frac{\left|\delta_{1}{ }^{(1)} \delta_{4}{ }^{(1)}\right|}{1-\left|\delta_{4}{ }^{(1)}\right|}\right]^{-1}\left\{| a _ { 1 } { } ^ { ( 1 ) } | \left(1+\left|\delta_{1}{ }^{(1)}\right|\right.\right.} \\
&+\left.\frac{\left|\delta_{1}{ }^{(1)} \delta_{4}{ }^{(1)}\right|}{1-\left|\delta_{4}{ }^{(1)}\right|}\right)+\left|b_{1}{ }^{(1)}\right|\left[\left|\delta_{2}{ }^{(1)}\right|\right. \\
&\left.+\left|\delta_{4}{ }^{(1)}\right| \frac{1+\left|\delta_{2}{ }^{(1)}\right|}{1-\left|\delta_{4}{ }^{(1)}\right|}\right] \\
&\left.+\left|\delta_{5}{ }^{(1)}\right|\left(1+\frac{\left|\delta_{4}{ }^{(1)}\right|}{1-\left|\delta_{4}{ }^{(1)}\right|}\right)\right] \leqq a_{2}{ }^{(2)}
\end{aligned}
$$

Similarly

$$
\left|b_{2}^{(1)}\right| \leqq b_{2}^{(2)}
$$


From (2.44) and (2.45) it is clear that

$$
\begin{aligned}
\left|W^{(1)}(x+h, y+h)\right| \leqq\left|W^{(1)}(x, y)\right|+\left|a_{1}^{(1)}\right|+\mid & b_{(2)}^{(1)} \mid \\
& \leqq W^{(2)}(x+h, y+h)
\end{aligned}
$$

and finally $\left|W_{X Y}^{(1)}\right| \leqq W_{X Y}^{(2)}$ follows from the difference equation (2.29).

3. Bounds for the Discretization Error. In Theorem 3.1 we shall obtain an explicit bound on the discretization error in terms of the data and certain higher derivatives of the solution. Here corollaries 1 and 2 of Theorem 2.1 form the basis for the error estimate. Following this we show how to bound the higher derivatives of $u(x, y)$ in terms of data.

TheOREM 3.1. Let $u(x, y)$ be the solution of (2.1) and (2.2) with $\varphi(x)=\psi(y)=0$ and let $U(x, y)$ be the solution of (2.9) and (2.10). Define

$$
\begin{aligned}
& K_{1}(\xi, \eta)=\max _{x \varepsilon[0, \xi], y \in[0, \eta]}|a(x, y)| \\
& K_{2}(\xi, \eta)=\max _{x \varepsilon[0, \xi], y \varepsilon[0, \eta]}|b(x, y)| \\
& K_{3}(\xi, \eta)=\max _{x \varepsilon[0, \xi], y \varepsilon[0, \eta]}|c(x, y)| \\
& K_{4}(\xi, \eta) \geqq \max _{x \varepsilon[0, \xi], y \varepsilon[0, n]}\left|L_{h} u(x, y)\right| .
\end{aligned}
$$

Then the discretization error $E(x, y)=u(x, y)-U(x, y)$. has the bound

$$
|E(\xi, \eta)| \leqq K_{4}(\xi, \eta) \int_{0}^{\xi} d x \int_{0}^{\eta} d y\left[e_{h} \sqrt{c_{5}}\{(\xi-x)+(\eta-y)\}\right]\left[e_{h}{ }^{K_{2}(\xi, \eta)(\xi-x)+K_{1}(\xi, \eta)(y-\eta)}\right]
$$

where $K_{4}(\xi, \eta)$ is given by (3.5). For $0 \leqq \bar{x} \leqq \xi, 0 \leqq \bar{y} \leqq \eta$ the bound for $|E(\bar{x}, \bar{y})|$ is given by (3.2).

Proof.

By Corollary 1 and Corollary 2 of Theorem 2.1 we see that (3.2) is valid for any $K_{4}$ which satisfies (3.1).

Before obtaining the bound (3.5) for $K_{4}(\xi, \eta)$ we introduce the notation

$$
\varphi_{M}=\max _{x \varepsilon[0, \xi], y \varepsilon[0, \eta]} \varphi(x, y) \quad \varphi_{m}=\min _{x \varepsilon[0, \xi], y \varepsilon[0, \eta]} \varphi(x, y)
$$

for an arbitrary function $(x, y)$. Let $x=m h-\frac{1}{2} h, y=n h-\frac{1}{2} h$, then by Taylor's theorem

$$
\begin{aligned}
& \left|u(x, y)_{X Y}-u(x, y)_{x y}\right| \leqq\left(\frac{1}{2} h\right)^{2} \frac{1}{4 !}\left\{\left|u_{x x x x}\right|_{M}+4\left|u_{x x x x}\right|_{M}+6\left|u_{x x y y}\right|_{M}\right. \\
& \left.+4\left|u_{x y y y}\right|_{M}+\left|u_{y y y}\right|_{M}\right\}=\alpha_{1} h^{2} \\
& \left|u(x, y)_{X}-u(x, y)_{x}\right| \leqq \frac{1}{4} h^{2}\left\{\left|u_{x x x}\right|_{M}+3\left|u_{x x y}\right|_{M}+3\left|u_{x y y}\right|_{M}\right. \\
& \left.+\left|u_{y y y}\right|_{M}\right\}=h^{2} \alpha_{2}, \\
& \left|u(x, y)_{Y}-u(x, y)_{\nu}\right| \leqq h^{2} \alpha_{2}, \\
& \mid \frac{1}{4}\left\{u\left(x-\frac{1}{2} h, y-\frac{1}{2} h\right)+u\left(x-\frac{1}{2} h, y+\frac{1}{2} h\right)\right. \\
& \left.+a\left(x+\frac{1}{2} h, y-\frac{1}{2} h\right)+u\left(x+\frac{1}{2} h, y+\frac{1}{2} h\right)\right\}-u(x, y) \leqq \frac{1}{8} h^{2}\left\{\left|u_{x x}\right|_{M}\right. \\
& \left.+2\left|u_{x y}\right|_{M}+\left|u_{y y}\right|_{M}\right\}=h^{2} \alpha_{3} .
\end{aligned}
$$


Certain of the derivatives on the right side of (3.4) can be replaced by $O\left(h^{2}\right)$ terms which involve even higher derivatives of $U(x, y)$. It is now clear that

$$
\begin{aligned}
\left|L_{h} u-L u\right| \leqq h^{2}\left\{\alpha_{1}+\left(|a|_{\mu}+\right.\right. & \left.|b|_{\mu}\right) \alpha_{2}+|c|_{\mu} \alpha_{3} \\
& \left.+\frac{1}{8}\left(\left|a_{x x}\right|_{\mu}\left|u_{x}\right|_{\mu}+\left|b_{y y}\right|_{\mu}\left|u_{\nu}\right|_{\mu}\right)\right\} .
\end{aligned}
$$

In view of the definition of $\alpha_{i}$ all that remains is to obtain bounds for the higher derivatives of $u(x, y)$. These will now be bounded starting with the lowest order.

From Frank \& v. Mises $[17 ;$ p. 812] and the method of majorants we see that the Riemann's function of the majorizing problem is given by

$$
\begin{aligned}
& V(x, y ; \xi, \eta)=I_{0}(2 \sqrt{\beta(\xi-x)(\eta-y)}) e^{|b|_{M}(\xi-x)+|a|_{M}(\eta-y)} \\
& \qquad \beta=|a|_{M}|b|_{M}+|c|_{M} .
\end{aligned}
$$

Hence we have the inequalities

$$
\begin{aligned}
& |u|_{M} \leqq \int_{0}^{\xi} d x \int_{0}^{\eta} d y V(x, y ; \xi, \eta)|f(x, y)| \\
& \left|u_{x}\right|_{M} \leqq \int_{0}^{\eta} d y V(\xi, y ; \xi, \eta)|f(\xi, y)|+\int_{0}^{\xi} d x \int_{0}^{\eta} d y V_{\xi}(x, y ; \xi, \eta)|f(x, y)| \\
& \left|u_{y}\right|_{M} \leqq \int_{0}^{\xi} d x V(x, \eta ; \xi, \eta)|f(x, \eta)|+\int_{0}^{\xi} d x \int_{0}^{\eta} d y V_{\eta}(x, y ; \xi, \eta)|f(x, y)| \\
& \left|u_{x y}\right|_{M} \leqq|a|_{M}\left|u_{x}\right|_{M}+|b|_{M}\left|u_{\nu}\right|_{M}+|c|_{M}|u|_{M}+|f|_{M} .
\end{aligned}
$$

The functions $u_{x x}$ and $u_{y y}$ are bounded as follows. We write the differential equation 2.1 in the form

$$
\begin{aligned}
& u_{x y}+a u_{x}=f-b u_{y}-c u \equiv g . \\
& u_{x y}+b u_{y}=f-a u_{x}-c u \equiv l .
\end{aligned}
$$

The left side of $(3.8, a)$ can be integrated with respect to $y$ by using the integrating factor

$$
e^{p(\xi, \eta, y)} \equiv \exp \left[\int_{y}^{\eta} a(\xi, \lambda) d \lambda\right]
$$

This together with the assumption that $u_{x}(x, 0)=0$ yields

$$
u_{\xi}(\xi, \eta)=\int_{0}^{\eta} g(\xi, y) e^{p(\xi, \eta, y)} d y
$$

We have similar integral representation for $u_{\eta}(\xi, \eta)$. Differentiation of (3.10) yields

$$
u_{\xi \xi}(\xi, \eta)=\int_{0}^{\eta}\left\{g(\xi, y) p_{\xi}(\xi, \eta, y)+g_{\xi}(\xi, y)\right\} e^{p} d y
$$

By repeating this process for successively higher derivatives and making use of the integral representations for lower order derivatives, we can express the derivatives of all orders in terms of the data. We can then apply well known inequalities to these representations to obtain computable bounds for $\alpha_{1}, \alpha_{2}, \alpha_{3}$, in (3.4).

We conclude this section with explicit bounds on the error between the first and 
the second order mixed derivatives of $u(x, y)$ and their finite difference counterparts of $U(x, y)$.

We see that:

$$
\begin{aligned}
& \left|u_{x}(P)-U_{X}(P)\right| \leqq\left|E(P)_{X}\right|+\left|u_{x}(P)-u(P)_{X}\right| \\
& \left|u_{y}(P)-U(P)_{Y}\right| \leqq\left|E(P)_{Y}\right|+\left|u_{y}(P)-u(P)_{Y}\right| \\
& \left|u_{x y}(P)-U(P)_{X Y}\right| \leqq\left|E(P)_{X Y}\right|+\left|u_{x y}(P)-u(P)_{X Y}\right| .
\end{aligned}
$$

The $O\left(h^{2}\right)$ bounds for $\left|u_{x}(P)-u(P)_{X}\right|,\left|u_{y}(P)-u(P)_{Y}\right|$, and $\mid u_{x y}(P)-$ $u(P)_{X Y} \mid$, in terms of higher derivatives of $u(x, y)$ are given by $h^{2} \alpha_{2}, h^{2} \alpha_{2}$ and $h^{2} \alpha_{1}$ respectively, where $\alpha_{2}$ and $\alpha_{1}$ are defined by (3.4).

To bound $\left|E(P)_{X}\right|,\left|E(P)_{Y}\right|$ and $\left|E(P)_{X Y}\right|$, we consider the boundary value problem

$$
\begin{gathered}
E_{X Y}=-A E_{x}-B E_{Y}-C E+\sigma(x, y) \\
E(x, 0)=E(0, y)=0
\end{gathered}
$$

where $\sigma(x, y)=L^{h} u-L u$ and $|\sigma(x, y)| \leqq K_{5}$, where $K_{5}$ denotes the righthand side of (3.5).

From Corollary 2 of section 2 it follows that:

$$
\begin{array}{ll}
|E(x, y)| \leqq E^{*}(x, y), & \left|E(x, y)_{X}\right| \leqq E^{*}(x, y)_{X}, \\
\left|E(x, y)_{Y}\right| \leqq E^{*}(x, y)_{Y}, & \left|E(x, y)_{X Y}\right| \leqq E^{*}(x, y)_{X Y}
\end{array}
$$

where $E^{*}(x, y)$ is the solution of the boundary value problem.

$$
\begin{gathered}
E^{*}(x, y)_{X Y}=C_{1} E_{X}^{*}(x, y)+C_{2} E^{*}(x, y)_{Y}+C_{3} E^{*}(x, y)+K_{5}, \\
E^{*}(x, 0)=E^{*}(0, y)=0
\end{gathered}
$$

with $C_{1}=\max _{x, y \varepsilon R}|A(x, y)|, C_{2}=\max _{x, y \varepsilon R}|B(x, y)|$, and $C_{3}=\max _{x, y \varepsilon R}|C(x, y)|$. By the transformation

$$
E^{*}(x, y)=\bar{E}(x, y) e_{h}{ }^{c_{2} x+c_{1} y},
$$

(3.15) reduces to

$$
\begin{gathered}
\bar{E}_{X Y}(x, y)=C_{5} \bar{E}(x, y)+K_{5} e_{h}^{-\left(C_{2} x+C_{1} y\right)} \\
\bar{E}(x, 0)=\bar{E}(0, y)=0
\end{gathered}
$$

with $C_{5}=C_{1} C_{2}+C_{3}$.

Denoting by $\bar{V}(\bar{x}, \bar{y}, x, y)$ the Riemann function associated with (3.17) and making use of the symmetry property of the Riemann function $[2$, p. 454] we have

From (2.41) we have

$$
\begin{aligned}
\bar{V}(\bar{x}, \bar{y} ; x, y)_{X Y} & =C_{5} \bar{V}(\bar{x}, \bar{y} ; x, y) \\
\bar{V}(\bar{x}, \bar{y} ; \bar{x}, y) & =\bar{V}(\bar{x}, \bar{y} ; x, \bar{y})=1 .
\end{aligned}
$$

$$
\bar{V}(\bar{x}, \bar{y} ; x, y) \leqq e_{h}^{\sqrt{\bar{c}_{5}}[(x-\bar{x})+(y-\bar{y})]} .
$$

Now let $z(\bar{x}, \bar{y} ; x, y)=e_{h}{ }^{\sqrt{C_{5}}[(x-\bar{x})+(y-\bar{y})]}$, and $\bar{z}=z-\bar{V}(\bar{x}, \bar{y}, x, y)$. It is easily 
seen that

$$
\begin{gathered}
\bar{z}(\bar{x}, \bar{y} ; x, y)_{X Y}=C_{5} \bar{z}(\bar{x}, \bar{y} ; x, y) \\
\bar{z}(\bar{x}, \bar{y} ; \bar{x}, y)=e_{h}^{\sqrt{c_{b}(y-\bar{y})}}-1, \bar{z}(\bar{x}, \bar{y}, x, \bar{y})=e_{h}^{\sqrt{\bar{c}_{b}(x-\bar{x})}}-1 .
\end{gathered}
$$

From (3.20) and Theorem 2.1, it follows that

$$
\bar{z}, \bar{z}_{X}, \bar{z}_{Y}, \bar{z}_{X Y} \geqq 0,
$$

and hence

$$
\begin{aligned}
\bar{V}(\bar{x}, \bar{y} ; x, y) & \leqq e_{h}^{\sqrt{C_{5}}(x-\bar{x}+y-\bar{y})} \\
\bar{V}(\bar{x}, \bar{y} ; x, y)_{X} & \leqq \sqrt{C_{5}} e_{h}^{\sqrt{C_{5}}(x-\bar{x}+y-\bar{y})} \\
\bar{V}(\bar{x}, \bar{y} ; x, y)_{Y} & \leqq \sqrt{C_{5}} e_{h}^{\sqrt{C_{5}}(x-\bar{x}+y-\bar{y})} \\
V(\bar{x}, \bar{y} ; x, y)_{X Y} & \leqq C_{5} e_{h}^{\sqrt{c_{5}}(x-\bar{x}+y-\bar{y})}
\end{aligned}
$$

Now by (2.18), $\bar{E}(x, y), \bar{E}(x, y)_{x}, \bar{E}(x, y)_{Y}$, and $\bar{E}(x, y)_{X Y}$ may be written as

$$
\begin{aligned}
& \bar{E}(x, y)=K_{5} \int_{0}^{x} d \mu \int_{0}^{\nu} d \nu \bar{V}(\bar{x}, \bar{y} ; x, y) e^{-\left(c_{2} x+c_{1} \bar{y}\right)} \\
& \bar{E}(x, y)_{x}=K_{5}\left[\int_{0}^{y} d \nu \bar{V}(x, \bar{y} ; x, y) e^{-\left(c_{2} x+c_{1} \bar{y}\right)}\right. \\
& \left.+\int_{0}^{x} d \mu \int_{0}^{y} d \nu \bar{V}(\bar{x}, \bar{y} ; x, y)_{x} e^{-\left(c_{2} \bar{x}+c_{1} \bar{y}\right)}\right] \\
& \bar{E}(x, y)_{Y}=K_{5}\left[\int_{0}^{x} d \mu \bar{V}(\bar{x}, y ; x, y) e^{-\left(c_{2} \bar{x}+c_{1} \bar{y}\right)}\right. \\
& \left.+\int_{0}^{x} d \mu \int_{0}^{y} d \nu \bar{V}(\bar{x}, \bar{y} ; c, y)_{Y} e^{-\left(C_{2} \bar{x}+c_{1} \bar{y}\right)}\right] \\
& \bar{E}(x, y)_{X Y}=K_{5} e_{h}^{-\left(c_{2} x+c_{1} y\right)}+C_{5} \bar{E}(x, y) .
\end{aligned}
$$

From (3.21) and (3.22) it follows that

$$
\begin{aligned}
& \bar{E}(x, y) \leqq K_{5} \int_{0}^{x} d \mu \int_{0}^{y} d \nu e_{h}^{\sqrt{c_{5}}(x-\bar{x}+y-\tilde{y})} e_{h}^{-\left(c_{2} \bar{x}+c_{1} \tilde{y}\right)}=\beta_{1} \\
& \bar{E}_{x}(x, y) \leqq K_{5} \int_{0}^{y} d \nu e_{h}^{-\left[c_{2} x+c_{1} \bar{y}\right]}+\sqrt{C_{5}} \int_{0}^{x} d \mu \int_{0}^{y} d \nu e_{h} \sqrt{\bar{C}_{5}(x-\bar{x}+y-\bar{y})} e_{h}^{-\left(C_{2} \bar{x}+C_{1} \bar{y}\right)}=\beta_{2} \\
& \bar{E}_{Y}(x, y) \leqq K_{5} \int_{0}^{x} d \mu e_{h}^{-\left[c_{2} \bar{x}+c_{1} y\right]}+\sqrt{C_{5}} \int_{0}^{x} d \mu \int_{0}^{y} d \nu e_{h} \sqrt{\bar{c}_{5}(x-\bar{x}+y-\bar{y})} e_{h}^{-\left(c_{2} \bar{x}+c_{1} \bar{y}\right)}=\beta_{3} \\
& \bar{E}(x, y)_{X Y} \leqq K_{5} e_{h}^{-\left[C_{2} x+c_{1} y\right]}+C_{5} \beta_{1} \text {. }
\end{aligned}
$$

$$
\begin{aligned}
& \text { Thus from (3.14) and (3.16) we obtain } \\
& \qquad \begin{aligned}
|E(x, y)| & \leqq E^{*}(x, y) \leqq \beta_{1} e^{c_{2} x+C_{1} y}=\gamma_{1} \\
\left|E(x, y)_{X}\right| & \leqq E^{*}(x, y)_{X} \leqq e_{h}{ }^{C_{2} x+C_{1} y}\left[\beta_{2}+C_{2} \beta_{1}\right]=\gamma_{2} \\
\left|E(x, y)_{Y}\right| & \leqq E^{*}(x, y)_{Y} \leqq e_{h}{ }^{C_{2} x+C_{1} y}\left[\beta_{3}+C_{1} \beta_{1}\right]=\gamma_{3} \\
\left|E(x, y)_{X Y}\right| & \leqq E^{*}(x, y)_{X Y} \leqq K_{5}+e_{h}{ }^{c_{2} x+C_{1} y}\left[C_{5} \beta_{1}+C_{1} \beta_{2}+C_{1} C_{2} \beta_{1}+C_{2} \beta_{3}\right] \\
& =\gamma_{4}
\end{aligned}
\end{aligned}
$$


Now substitution of the bounds in (3.23) in (3.12) yields

$$
\begin{gathered}
\left|u_{x}(P)-U(P)_{x}\right| \leqq \gamma_{2}+h^{2} \alpha_{2}, \quad\left|u_{y}(P)-U(P)_{Y}\right| \leqq \gamma_{3}+h^{2} \alpha_{2}, \\
\left|u_{x y}(P)-U(P)_{X Y}\right| \leqq \gamma_{4}+h^{2} \alpha_{1} .
\end{gathered}
$$

4. General Quasi-Linear Case. In this section we shall consider a method for approximating the solution of

$$
\begin{array}{cr}
u_{x y}=f(x, y, u, v, w), & (x, y) \in R \\
v=u_{x}, \quad w=u_{y} & \\
u(x, 0)=u(0, y)=0 . &
\end{array}
$$

We assume that $f(x, y, u, v, w)$ satisfies the Lipschitz condition

$$
\begin{aligned}
& |f(x, y, \bar{u}, \bar{v}, \bar{w})-f(x, y, u, v, w)| \\
& \quad \leqq K_{1}|\bar{u}-u|+K_{2}|\bar{v}-v|+K_{3}|\bar{w}-w|,
\end{aligned}
$$

and is bounded for

$$
-\infty<u, v, w<\infty .
$$

Under these conditions it is known [Diaz, 6] that (4.1) possesses a unique solution.

We introduce a network as before and define the difference quotients as in (2.4). Again we extend the domain of definition of an arbitrary mesh function to the centers of squares as averages so that the chain rule for differencing (2.6) is valid. We define the finite difference analogue of (4.1) to be

$$
\begin{aligned}
& U_{X Y}(P)=f\left(P, U(P), U_{X}(P), U_{Y}(P)\right), \\
& P=\left(m h+\frac{1}{2} h, n h+\frac{1}{2} h\right) \quad m, n=0,1,2,3, \cdots \\
& U(x, 0)=U(0, y)=0 .
\end{aligned}
$$

At each square with $P$ given in (4.3) the difference equation becomes

$$
\begin{aligned}
U[(m+1) h,(n+1) h]=U & (m h, n h) \\
& +h\left[U_{X}\left(m h+\frac{1}{2} h, n h\right)+U_{Y}\left(m h, n h+\frac{1}{2} h\right)\right] \\
& +h^{2} f\left[P, U(P), U_{X}(P), U_{Y}(P)\right] .
\end{aligned}
$$

Then (4.4) is of the form

$$
U(m h, n h)=g[U(m h, n h)]
$$

where from (4.2) $g$ is a continuous function of $U(m h, n h)$. If we assume that $h$ is so small that

$$
\frac{1}{2} h\left[K_{2}+K_{3}+\frac{1}{2} h K_{1}\right]<1
$$

then by the contraction mapping theorem we can solve (4.4) for $U[(m+1) h$, $(n+1) h]$ in terms of $U[(m+1) h, n h], U[m h,(n+1) h]$, and $U(m h, n h)$, through an iterative process.

The discretization error $E(P)=u(P)-U(P)$ satisfies the difference inequality 


$$
\begin{aligned}
& E_{X Y} \leqq K_{1}|E|+K_{2}\left|E_{X}\right|+K_{3}\left|E_{Y}\right|+K_{4} \\
& K_{4}=K_{1}|u-\bar{u}|_{M}+K_{2}\left|u_{x}-u_{X}\right|_{\mu} \\
& \quad+K_{3}\left|u_{y}-u_{Y}\right|_{\mu}+\left|u_{x y}-u_{X Y}\right|_{\mu}
\end{aligned}
$$

at points $P=\left(m h+\frac{1}{2} h, n h+\frac{1}{2} h\right)$ with

$$
\begin{aligned}
\bar{u}(P)=\frac{1}{4}\{u[(m+1) h,(n+1) h]+ & u[(m+1) h, n h] \\
& +u[m h,(n+1) h]+u(m h, n h)\}
\end{aligned}
$$

In addition we note that

$$
E(x, 0)=E(0, y)=0 .
$$

We pose the majorizing problem

$$
\begin{gathered}
W_{X Y}=K_{1} W+K_{2} W_{x}+K_{3} W_{Y}+K_{4} \\
W(x, 0)=W(0, y)=0 .
\end{gathered}
$$

By an obvious modification of the proof of Theorem 2.1, Corollary 2 we conclude that

$$
|E| \leqq W, \quad\left|E_{X}\right| \leqq W_{X}, \quad\left|E_{Y}\right| \leqq W_{Y}, \quad\left|E_{X Y}\right| \leqq W_{X Y} .
$$

The following theorem gives the bound on the discretization error in this case.

Theorem 4.1. Let $E=u-U$ where $u$ and $U$ are the solutions of (4.1) and (4.3) respectively. Then $E$ has the bound

$$
|E| \leqq K_{4} \int_{0}^{\xi} d x \int_{0}^{\eta} d y\left[e_{h}{ }^{\sqrt{C_{5}}\{(\xi-x)+(\eta-y)\}}\right] e_{h}{ }^{K_{3}(\xi-x)+K_{2}(\eta-y)}
$$

where $C_{5}$ is given by

$$
C_{5}=K_{1}+K_{2} K_{3} \text {. }
$$

Proof: The theorem follows from (4.9) and Theorem 2.1, Corollary 1.

The bound (4.10) on the discretization error will be explicit once we show how to bound $K_{4}$ in terms of the data of the problem. At this point we assume that the partial derivatives of $f$ with respect to $u, v$, and $w$ through the third order are bounded. The quantity $K_{4}$ in (4.7) can be bounded in terms of the higher derivatives of $u(x, y)$ as is seen from (3.4). Now if $|f|_{M}=|f(x, y, 0,0,0)|_{M}$ and $v(x, y)$ is a solution of

$$
\begin{gathered}
v_{x y}=K_{1} v+K_{2} v_{x}+K_{3} v_{y}+|f|_{\mu} \\
v(x, 0)=v(0, y)=0
\end{gathered}
$$

then by the method of successive approximations we see that

$$
|u| \leqq v, \quad\left|u_{x}\right| \leqq v_{x}, \quad\left|u_{y}\right| \leqq v_{y}, \quad\left|u_{x y}\right| \leqq v_{x y} .
$$

Hence we see that bounds for $u, u_{x}, u_{y}, u_{x y}$, are given by (3.7) where we make the substitutions

$$
|a|_{M}=K_{2}, \quad|b|_{M}=K_{3}, \quad \beta=K_{1}+K_{2} K_{3} .
$$


Bounds for the higher mixed derivatives in terms of the known bounds for $|u|$, $\left|u_{x}\right|,\left|u_{y}\right|,\left|u_{x y}\right|$ are obtained by differentiating the differential equation (4.1), as was done in section 3 . To obtain bounds for the higher pure derivatives we use a variation of the method applied in section 3. We illustrate the method for $u_{x x}$.

From (4.1) we see that

$$
u_{x x y}=f_{x}+f_{u} u_{x}+f_{v} u_{x x}+f_{w} u_{x y} .
$$

Hence by introducing an integrating factor

$$
u_{x x}(x, y)=\int_{0}^{y} d \eta g(x, \eta) \exp \left[-\int_{\eta}^{y} l(x, \lambda) d \lambda\right]
$$

where

$$
\begin{aligned}
g(x, y) & =f_{x}+f_{u} u_{x}+f_{w} u_{x y} \\
l(x, y) & =f_{v}\left[x, y, u(x, y), u_{x}(x, y), u_{y}(x, y)\right] .
\end{aligned}
$$

Now under the assumption that $f_{x}, f_{u}, f_{v}, f_{w}$ are bounded we can obtain an explicit bound for $u_{x x}$. The bounds thus obtained for the higher derivatives allow us to bound $K_{i}$ in (4.10).

5. An example. Consider the boundary value problem:

$$
\begin{gathered}
u_{x y}(x, y)-x y u(x, y)=x^{2} y^{2}\left(9-x^{2} y^{2}\right) \\
u(x, 0)=u(0, y)=0
\end{gathered}
$$

in the closed square $s:\{0 \leqq x \leqq 1,0 \leqq y \leqq 1\}$, which has the solution $u=x^{3} y^{3}$. From (3.7) we have

$$
\begin{aligned}
|u(\xi, \eta)|_{M} \leqq & \int_{0}^{\xi} \int_{0}^{\eta}|v(x, y ; \xi, \eta)|\left(9-x^{2} y^{2}\right) x^{2} y^{2} d y d x \\
\left|u(\xi, \eta)_{\xi}\right|_{M} \leqq \int_{0}^{\eta}|v(\xi, y ; \xi, \eta)| & \left(9-\xi^{2} y^{2}\right) d y d x \\
& +\int_{0}^{\xi} \int_{0}^{\eta}\left|v_{\xi}(x, y ; \xi, \eta)\right|\left(9-x^{2} y^{2}\right) x^{2} y^{2} d y d x \\
\left|u(\xi, \eta)_{\xi}\right|_{M} \leqq \int_{0}^{\xi}|v(x, \eta ; \xi, \eta)|\left(9-x^{2} \eta^{2}\right) x^{2} \xi^{2} d x & +\int_{0}^{\xi} \int_{0}^{\eta}\left|v_{\eta}(x, y ; \xi, \eta)\right|\left(9-x^{2} y^{2}\right) x^{2} y^{2} d y d x
\end{aligned}
$$

where

$$
\begin{aligned}
v(x, y ; \xi, \eta) & =I_{0}\left[\sqrt{|x y|_{\eta}(\xi-x)(\eta-y)}\right] \leqq I_{0}(2) \approx 2.28 \\
v(\xi, y ; \xi, \eta) & =v(x, \eta ; \xi, y)=1 \\
v_{\xi}(x, y ; \xi, \eta) & =\frac{\sqrt{\eta-y}}{\sqrt{\xi-x}} I_{1}\left[2 \sqrt{|x y|_{\eta}(\xi-x)(\eta-y)}\right] \\
& \leqq \frac{\sqrt{\eta-y}}{\sqrt{\xi-x}} I_{1}(2) \approx 1.6 \frac{\sqrt{\eta-y}}{\sqrt{\xi-x}}
\end{aligned}
$$




$$
v_{\eta}(x, y ; \xi, \eta) \leqq \frac{\sqrt{\xi-x}}{\sqrt{\eta-y}} I_{1}(2) \approx 1.6 \frac{\sqrt{\xi-x}}{\sqrt{\eta-y}}
$$

From (5.2) and (5.3) by integration it follows that

$$
\begin{aligned}
& |u| \leqq 2.18 ; \quad\left|u_{x}\right|,\left|u_{y}\right| \leqq 5.36 \\
& \left|u_{x y}\right| \leqq 11.18 ; \quad\left|u_{x x}\right|,\left|u_{y y}\right| \leqq 25.54 \\
& \left|u_{x x y}\right|,\left|u_{x y y}\right| \leqq 25.54 ; \quad\left|u_{x x x}\right|,\left|u_{y y y}\right| \leqq 54.26 \\
& \left|u_{x x x y}\right|,\left|u_{x y y y}\right| \leqq 54.26 ; \quad\left|u_{x x y y}\right| \leqq 59.08 \\
& \left|u_{x x x x}\right|,\left|u_{y y y y}\right| \leqq 148.88
\end{aligned}
$$

Now $K_{4}(1,1)$ appearing in $(3.2)$ is given by

$$
K_{4}(1,1) \leqq h^{2}\left(\alpha_{1}+\alpha_{3}\right)
$$

where $\alpha_{1}$ and $\alpha_{3}$ are given by (3.4). We have $\alpha_{1}=11.36, \alpha_{3}=9.18$, thus from (3.2) we obtain

$$
|E|_{M} \leqq 20.54 h^{2} \int_{0}^{\xi} \int_{0}^{\xi} e_{\eta} \sqrt{|x y|_{\eta}\{(\xi-x)+(\eta-y)\}} d x d y \leqq 60.59 h^{2}
$$

The solution to the finite difference analog of (5.1) was obtained on the computer at the U. S. Naval Ordnance Laboratory. The calculations were made with $h=0.1$ with the results given in the following table.

\begin{tabular}{l|r|r|r|r}
\hline$x$ & $y$ & \multicolumn{1}{c|}{$u(x, y)$} & $U(x, y)$ & $E=u-U$ \\
\cline { 2 - 4 } .2 & .2 & $6.40 \times 10^{-5}$ & $6.392 \times 10^{-5}$ & $8.0 \times 10^{-8}$ \\
.2 & .4 & $5.12 \times 10^{-4}$ & $5.116 \times 10^{-4}$ & $4.0 \times 10^{-7}$ \\
.2 & .6 & $1.728 \times 10^{-3}$ & $1.727 \times 10^{-3}$ & $1.2 \times 10^{-6}$ \\
.2 & .8 & $4.096 \times 10^{-3}$ & $4.093 \times 10^{-3}$ & $2.7 \times 10^{-6}$ \\
.2 & 1.0 & $8.0 \times 10^{-3}$ & $7.995 \times 10^{-3}$ & $5.2 \times 10^{-6}$ \\
.4 & .4 & $4.096 \times 10^{-3}$ & $4.095 \times 10^{-3}$ & $1.3 \times 10^{-6}$ \\
.4 & .6 & $1.3824 \times 10^{-2}$ & $1.3821 \times 10^{-2}$ & $3.2 \times 10^{-6}$ \\
.4 & .8 & $3.2768 \times 10^{-2}$ & $3.2762 \times 10^{-2}$ & $6.5 \times 10^{-6}$ \\
.4 & 1.0 & $6.4 \times 10^{-2}$ & $6.3988 \times 10^{-2}$ & $1.2 \times 10^{-5}$ \\
.6 & .6 & $4.6656 \times 10^{-2}$ & $4.6649 \times 10^{-2}$ & $6.7 \times 10^{-6}$ \\
.6 & .8 & $1.1059 \times 10^{-1}$ & $1.1059 \times 10^{-1}$ & $1.3 \times 10^{-5}$ \\
.6 & 1.0 & $2.16 \times 10^{-1}$ & $2.1598 \times 10^{-1}$ & $2.2 \times 10^{-5}$ \\
.8 & .8 & $2.6214 \times 10^{-1}$ & $2.6212 \times 10^{-1}$ & $2.2 \times 10^{-5}$ \\
.8 & 1.0 & $5.12 \times 10^{-1}$ & $5.1196 \times 10^{-1}$ & $3.7 \times 10^{-5}$ \\
1.0 & 1.0 & 1.0 & $9.9994 \times 10^{-1}$ & $6.2 \times 10^{-5}$ \\
\hline
\end{tabular}

In this case the $a$ priori bound on the discretization error given by 5.7 is $6 \times 10^{-3}$ and the observed maximum error in the table above is $6 \times 10^{-5}$. The fact that the error bound is 100 times too large is in part attributable to the crude manner in which the higher derivatives of $u(x, y)$ have been computed. It is clear that the higher derivatives should be solved for directly in terms of $u, u_{x}, u_{y}$ for the particular problem under consideration with inequalities obtained only at the last moment. 
We are indebted to Theodore Orlow and Al Johnson of the U. S. Naval Ordnance Laboratory for their kind assistance in the calculation of the above example.

Georgetown University

Washington D.C.

University of Maryland

College Park, Maryland

1. A. K. Azrz \& J. B. Diaz, "On linear hyperbolic equations with initial conditions on higher derivatives." (To appear.)

2. R. Courant \& D. Hilbert, Methods of Mathematical Physics, Vol. 2, Interscience, New York, 1962.

3. R. Courant, K. Friedrichs, \& H. Lewy, "Uber die partiellen Differentialgleichungen der mathematischen Physik," Math. Ann., v. 100, 1928, p. 32-74.

4. R. Courant, E. Isaacson, \& M. Rees, "On the solution of nonlinear hyperbolic differential equations by finite differences," Comm. Pure Appl. Math. v. 7, 1954, p. 345-391.

5. R. T. DAmes, "Stability and convergence for a numerical solution of the Goursat problem," J. Math. Physics, v. 38, 1959, p. 42-67.

6. J. Diaz, "On an analogue of Euler-Cauchy polygon method for the numerical solution of $u_{x y}=f\left(x, y, u, u_{x}, u_{y}\right)$," Arch. Rational Mech. Anal., v. 4, 1958, p. 357-390.

7. K. Friedrichs \& H. LEWY, "Das Anfangswertproblem einer beliebigen nichtlinearen hyperbolischen Differentialgleichung," Math. Ann., v. 99, 1928, p. 200-221.

8. G. Forsythe \& W. Wasow, Finite Difference Methods for Partial Differential Equations, John Wiley \& Sons, New York, 1960.

9. H. B. Keller, "On the solution of semi-linear hyperbolic systems by unconditionally stable methods," Comm. Pure Appl. Math., v. 14, 1961, p. 447-456.

10. P. D. LAx, "On the stability of difference approximations to solutions of hyperbolic equations with variable coefficients," Comm. Pure Appl. Math., v. 14, 1961, p. 497-520.

11. M. LEES, "Energy inequalities for the solution of differential equations," Trans. Amer. Math. Soc., v. 94,1960 , p. 58-73.

12. M, Lees, "The solution of positive-symmetric hyperbolic system by difference methods," Proc. Amer. Math. Soc., v. 12, 1961, p. 195-204.

13. R. MooRe, "A Runge-Kutta procedure for the Goursat problem in hyperbolic partial differential equations." Arch. Rat. Mech. Anal. v. 7, 1961, p. 37-63.

14. R. D. Richtmyer, "Difference methods for initial value problems," Interscience Tracts in Pure and Appl. Math., Vol. 4, Interscience publishers, New York, 1957.

15. H. J. STETter, "On the convergence of characteristic finite difference methods of high accuracy for quasi-linear hyperbolic equations," Num. Math., v. 3, 1961, p. 321-344.

16. V. Thомеe, "Difference methods for two-dimensional mixed problems for hyperbolic first order systems," Arch. Rational Mech. Anal., v. 8, 1961, p. 68-87.

17. Philipp Frank \& Richard von Mises, Differential-gleichungen der Physik, Vol. 1, Rosenberg, New York, 1943, p. 812.

18. H. WEINBERGER, "Exact bounds for solutions of hyperbolic equations by finite difference methods," Proceedings of the Rome Symposium On the Numerical Treatment of Partial Differential Equations with Real Characteristics, Rome, 1959, p. 88-97.

19. H. WEINBERGER, "Error bounds in finite-difference approximations to solutions of symmetric hyperbolic systems," J. Soc. Indust. Appl. Math., v. 7, 1959, p. 49-75.

20. B. H. WendRofF, "On central difference equations for hyperbolic systems," J. Soc. Indust. Appl. Math., v. 8, 1960, p. 549-555. 